\title{
Research of Aerial Hydrazine Fuel Guarantee Safety Assessment Based on Vague-SPA and Fuzzy Proximal Grade
}

\author{
Zongshun Hu, Zhijie Huang \\ Air Force Logistics College, Department of Aviation Four Stations, Xuzhou 221000, China
}

Keywords: aerial hydrazine fuel; safety assessment; fuzzy matter-element; Vague-SPA; fuzzy close degree

\begin{abstract}
Aiming at the problem of aerial hydrazine fuel guarantee safety assessment, an assessment model based on Vague-SPA and fuzzy close degree was proposed in accordance to the fuzzy matter-element. Firstly, based on the definition and actual situation, an assessment index system and fuzzy matter-element model of aerial hydrazine fuel guarantee were built. Furthermore, the weight was optimized by applying fuzzy close degree of the decision model. The authors built an assessment model with Vague-SPA clustering combined by using fuzzy mathematics and SPA analysis to reduce the influence of subjectivity during the evaluating process. Finally, objective and reasonable results were obtained through a practical example, and it proved the effectiveness of the method and provided a new way for the safe assessment in aerial hydrazine fuel guarantee.
\end{abstract}

\section{Introduction}

As an emergency response to sudden energy emergencies, Hydrazine is an excellent liquid propellant that can be rapidly decomposed under the action of a catalyst to provide emergency energy to the emergency hydraulic pump and emergency generator to obtain the emergency hydraulic pressure required by the aircraft energy and power. At the same time, hydrazine fuel is flammable, explosive and toxic, once the safety accident occurs, it will cause casualties and economic losses, and the surrounding environment causing serious pollution.

At present, the relevant scholars at home and abroad for the safety of hydrazine fuel security using Road Chemistry, Analytic Hierarchy Process and gray evaluation, fuzzy comprehensive evaluation, BP and Hopfield neural network and other evaluation methods [1-3]. These methods often need a large amount of original data to support, the lack of historical data lead to poor results of fitting; the same time, quantitative indicators in the quantitative treatment, weakening the correlation between the same level indicators, useful information is lost, can not reflect the evaluation object The same evaluation of the degree of fierce competition.

Based on the fuzzy matter-element theory, the index system of hydrazine fuel support is constructed. The vector of each attribute value and the angle cosine of the positive and negative ideal scheme are calculated by fuzzy proximity degree. The optimal value of each index is obtained by fuzzy close degree optimal solution. The optimal solution is introduced into the modified Vague-SPA clustering model as the modified weight, and the IDO correlation coefficient between the various sub-systems is calculated. Finally, the fuzzy similarity matrices derived from the transitive closure are used to filter the similarity of the scheme, Safety Evaluation of Hydrazine Fuel Support in the target Station.

\section{Basic theory}

\subsection{Fuzzy compound element}

The fuzzy matter element $K$ is written as: $K=(M, C, \mu)$, where $M$ is the thing; $C$ is the feature of describing things; $\mu(x)$ is the fuzzy membership of the corresponding value $x$ of the thing characteristic $C$ [4]. If there are $n$ things, the $i$-th thing is $M_{i}(i=1,2, \cdots, n)$; there are $m$ features, the $j$-th feature is written as $C_{j}(j=1,2, \cdots, N), \mu\left(x_{i j}\right)$ is the fuzzy value corresponding to the $j$-th item of 
the $i$-th thing, and the membership degree. The $m$-dimensional fuzzy compound matter element of $n$ things is expressed as:

$$
\underset{\sim}{K * m}=\left(\begin{array}{cccc} 
& C_{1} & \cdots & C_{m} \\
M_{1} & \mu\left(x_{11}\right) & \cdots & \mu\left(x_{1 m}\right) \\
\vdots & \vdots & \vdots & \vdots \\
M_{n} & \mu\left(x_{n 1}\right) & \cdots & \mu\left(x_{n m}\right)
\end{array}\right)
$$

2.2 Fuzzy clustering based on SPA

\subsubsection{Multivariate Connection Number Model}

Set pair analysis refers to the specific conditions of the study, the degree of association will be introduced with similarity or relevance of the set, the uncertainty of the dialectical analysis and mathematical processing methods [5]. The multiple connection number is a kind of hierarchical structure function which is derived from $U=A+B_{1} i_{1}+B_{2} i_{2}+\cdots B_{n} i_{n}+C j$, which is based on the expansion of $U=A+B i+C j$ on item $B i$. The relation $\mu(A)=a+b_{1} i_{1}+b_{2} i_{2}+c j$ between the set pair $H(X, Y)$ is given by $U=A+B_{1} i_{1}+B_{2} i_{2}+C j$.

Taking into account the weight of each feature difference, then $\mu(A)=a+b_{1} i_{1}+b_{2} i_{2}+c j=\sum_{k=1}^{S} \omega_{k}+\sum_{k=S+1}^{S+F_{1}} \omega_{k} i_{1}+\sum_{k=S+F_{1}+1}^{S+F_{1}+F_{2}} \omega_{k} i_{2}+\sum_{k=S+F_{1}+F_{2}+1}^{N} \omega_{k} j$. Where $\omega_{k}\left(k=1,2, \cdots, N ; \sum_{k=1}^{N} \omega_{k}=1\right)$ is the eigenvalue weight.

\subsubsection{SPA fuzzy clustering}

According to the basic principle of fuzzy set pair and clustering analysis, the similarity index which is derived from the same and different inverse vector is calculated by matrix. Specific steps are as follows [6]:

1) Set the classification subsystem $M$ of the system $M=\left\{M_{1}, M_{2}, \cdots, M_{n}\right\}$.

2) Select the standard subsystem. According to the research background, one subsystem is determined as a reference subsystem.

3) Calculate the IDO contact vector of the remaining $n-1$ subsystems relative to the reference subsystem. If $M_{1}$ is selected as the reference subsystem, and the feature number between $M_{1}$ and $M_{k}(k=2,3, \cdots, n)$ is $\mathrm{N}$, the set of $A_{k}$ and $A_{1}$ is defined. The IDO contact vector of the $t(t=1,2, \cdots, N)$ feature number is $R_{t}^{k}=\left(a_{t}^{k}, b_{t 1}^{k}, b_{t 2}^{k}, c_{t}^{k}\right)$, and $N$ factors affect the clustering mode of the subsystem And the factor weight $\omega=\left(\omega_{1}, \omega_{2}, \cdots, \omega_{N}\right), \sum_{i=1}^{N} \omega_{i}=1$ is obtained by fuzzy proximity degree method. Construct the multidimensional contact matrix $R^{k}=\left(R_{1}^{k}, R_{2}^{k}, \cdots, R_{N}^{k}\right)^{T}$, so the similarity and dissimilarity degree between the subsystem $M_{k}$ and the reference subsystem $M_{1}$ is:

$$
\eta^{k}=\omega \cdot R^{k} \cdot E=a^{k}+b_{1}^{k} i_{1}+b_{2}^{k} i_{2}+c^{k} j=\left(\omega_{1}, \omega_{2}, \cdots, \omega_{N}\right)\left(\begin{array}{cccc}
a_{1}^{k} & b_{11}^{k} & b_{12}^{k} & c_{1}^{k} \\
a_{2}^{k} & b_{21}^{k} & b_{22}^{k} & c_{2}^{k} \\
\vdots & \vdots & \vdots & \vdots \\
a_{N}^{k} & b_{N 1}^{k} & b_{N 2}^{k} & c_{N}^{k}
\end{array}\right)\left(\begin{array}{c}
1 \\
i_{1} \\
i_{2} \\
j
\end{array}\right)
$$

The vector is expressed as: $\eta^{k}\left(a^{k}, b_{1}^{k}, b_{2}^{k}, c^{k}\right)$.

4) Calculate the IDO correlation coefficient of each subsystem, and get the fuzzy similarity matrix. Two sub-systems $M_{l}$ and $M_{k}$ are set up, which are NL and NK for the similarity and dissimilarity relation vector with standard subsystem. Is defined

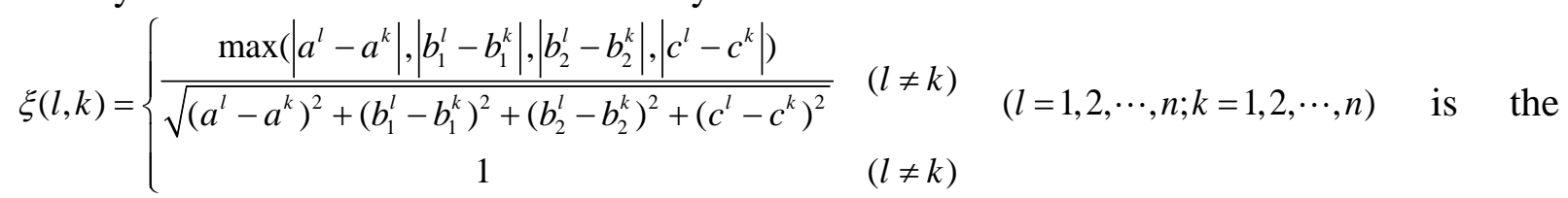

subsystem and the IDO correlation coefficient.

The similarity and dissimilarity correlation coefficient between the remaining subsystems is calculated, and the fuzzy similar symmetric matrix D is obtained, where 


$$
d_{l k}=\xi(l, k)=\xi(k, l)=d_{k l} \text {. }
$$

5) Fuzzy similarity matrix $D=\left(d_{l k}\right)_{n \times n}$, the calculated transitive closure $t(D)=D^{*}=\left(d_{l k}^{*}\right)_{n \times n}$.

6) Let $\lambda \in[0,1]$, fuzzy clustering of the subsystems, clustering $d_{l k}^{*} \geq \lambda$ subsystems into a class.

2.3 Fuzzy proximity

From the definition of fuzzy compound element, construct the decision matrix $K=\left(k_{i j}\right)_{n \times m}^{T}$, $\omega=\left(\omega_{1}, \omega_{2}, \cdots, \omega_{m}\right)$ is the attribute weight vector, where $\sum_{i=1}^{m} \omega_{i}=1 . H$ is the weight vector set of known weight information attributes. In order to eliminate the influence of different dimensions, the decision matrix is normalized and $K^{\prime}=\left(k_{i j}^{\prime}\right)_{n \times m}^{T}$ is obtained.

The aggregate attribute value of the transaction $M_{i}$ is $z_{i}(\omega)=\sum_{j=1}^{m} k_{i j}^{\prime} \omega_{j}$. Taking into account the uncertainty of the weight of each attribute, and for a given $\omega \in H$, the overall attribute value $z_{i}(\omega)$ bigger the better, the introduction of fuzzy proximity, the specific steps are as follows [7]:

1) Let $z_{i}^{\max }(\omega)=\sum_{j=1}^{m} k_{i j}^{\prime} \omega_{j}$ be the ideal value of the comprehensive property of things $M_{i}$, and there exists $\omega^{\prime} \in H$, such that $Z^{\prime}=\sqrt{\sum_{i=1}^{n}\left(z_{i}^{\max }\right)^{2}}$ is maximum. Similarly, let $z_{i}^{\min }(\omega)=\sum_{j=1}^{m} k_{i j}^{\prime} \omega_{j}$ be the negative ideal value of the comprehensive attribute of thing $M_{i}$, and there exists $\omega^{\prime} \in H$, such that $Z^{\prime}=\sqrt{\sum_{i=1}^{n}\left(z_{i}^{\max }\right)^{2}}$ is minimum.

2) The cosine of the integrated attribute value vector $z(\omega)=\left(z_{1}(\omega), z_{2}(\omega), \cdots, z_{n}(\omega)\right)$ and the ideal point $z^{\max }=\left(z_{1}^{\max }, z_{2}^{\max }, \cdots, z_{n}^{\max }\right)$ is $\cos \theta=\frac{\sum_{i=1}^{n} z_{i}^{\max } z_{i}(\omega)}{\sqrt{\sum_{i=1}^{n}\left(z_{i}^{\max }\right)^{2}} \sqrt{\sum_{i=1}^{n}\left(z_{i}(\omega)\right)^{2}}}$; Similarly, the angle cosine with the negative ideal point is $\cos \beta=\frac{\sum_{i=1}^{n} z_{i}^{\min } z_{i}(\omega)}{\sqrt{\sum_{i=1}^{n}\left(z_{i}^{\min }\right)^{2}} \sqrt{\sum_{i=1}^{n}\left(z_{i}(\omega)\right)^{2}}}$.

3) Define the fuzzy closeness function $s(\omega)=\frac{\cos \theta}{\cos \theta+\cos \beta}$. For any $\omega \in H$, the greater the value of $s(\omega)$, the closer to the ideal point, the closer to the optimal state.

Compared with the traditional target close degree, the fuzzy close degree can reflect the relation among the multiple objects, and effectively achieve the balance among the objects.

\section{Fuzzy safety assessment index system and fuzzy composite element}

An airport hydrazine fuel station is responsible for a flight group of hydrazine fuel security tasks, as managers of hydrazine fuel security status and development trend is not clear, so that it can not be a good security management, is to protect its work related to Safety assessment, analysis of security status and development trends, in order to facilitate the management of safety management. The structure of fuzzy compound matter-elements is shown in Table 1. 
Table 1 assessment index grades and standard of aerial stations

\begin{tabular}{ccccccc}
\hline & $\begin{array}{c}\text { Transport } \\
\text { module }\end{array}$ & $\begin{array}{c}\text { Laboratory } \\
\text { module }\end{array}$ & $\begin{array}{c}\text { Storage } \\
\text { module }\end{array}$ & $\begin{array}{c}\text { Filling } \\
\text { module }\end{array}$ & $\begin{array}{c}\text { Waste } \\
\text { disposal } \\
\text { module }\end{array}$ & $\begin{array}{c}\text { Field } \\
\text { protection } \\
\text { module }\end{array}$ \\
\hline $\begin{array}{c}\text { excellent } \\
\text { good }\end{array}$ & 85 & 80 & 90 & 85 & 90 & 85 \\
difference & 60 & 55 & 65 & 60 & 65 & 60 \\
\hline$M_{1}$ & 65 & 25 & 40 & 35 & 40 & 30 \\
$M_{2}$ & 68 & 70 & 74 & 84 & 92 & 82 \\
$M_{3}$ & 79 & 71 & 84 & 83 & 88 & 90 \\
$M_{4}$ & 90 & 84 & 76 & 84 & 78 & 89 \\
$M_{5}$ & 87 & 69 & 74 & 73 & 84 & 82 \\
$M_{6}$ & 86 & 80 & 85 & 94 & 70 & 78 \\
$M_{7}$ & 91 & 89 & 83 & 94 & 85 & 74 \\
$M_{8}$ & 87 & 84 & 82 & 79 & 87 & 91 \\
\hline
\end{tabular}

\section{Establishment of evaluation model based on Vague-SPA and fuzzy close degree}

The weight information of the known weight information attribute is: $H=\left\{\omega=\left(\omega_{1}, \omega_{2}, \cdots, \omega_{m}\right)\right\}$, $0.1 \leq \omega_{1} \leq 0.2, \quad 0.07 \leq \omega_{2} \leq 0.21, \quad 0.15 \leq \omega_{3} \leq 0.23, \quad 0.13 \leq \omega_{4} \leq 0.22, \quad 0.2 \leq \omega_{5} \leq 0.3, \quad 0.11 \leq \omega_{6} \leq 0.17$, $\sum_{i=1}^{m} \omega_{i}=1$

According to the above calculation steps, we call the lingo function to get the optimal solution $\omega^{*}=(0.1,0.15,0.15,0.13,0.3,0.17)$ and fuzzy close degree $s\left(\omega^{*}\right)=0.4994$.

From the structure of fuzzy compound matter, we can see that system $\mathrm{M}$ is composed of eight subsystems: $M=\left\{M_{1}, M_{2}, \cdots, M_{8}\right\}$.Determine $M_{1}$ as the reference subsystem $M_{1} \neq, 65707484,9282$.

Each station index score $M_{k}(k=2,3, \cdots, 8)$ and reference to the composition of the set of $M_{1}$ on the $t(t=1,2, \cdots, 6)$ characteristics of the IDO contact vector:

$$
\begin{array}{cc}
R_{1}^{2}=(0.96,0.007,0.010,0.023), & R_{2}^{2}=(0.93,0.012,0.004,0.054), \\
R_{3}^{2}=(0.88,0.023,0.008,0.089), & R_{4}^{2}=(0.88,0.001,0.001,0.008), \\
R_{5}^{2}=(0.96,0.017,0.014,0.009), & R_{6}^{2}=(0.91,0.035,0.01,0.045) 。
\end{array}
$$

According to the optimal solution weight, from 2.2.2 to obtain the subsystems and the reference system of the same and different anti-contact degree:

$$
\begin{array}{ll}
\eta^{2}=0.939+0.017 i_{1}+0.009 i_{2}+0.035 j, & \eta^{3}=0.906+0.011 i_{1}+0.012 i_{2}+0.07 j, \\
\eta^{4}=0.922+0.017 i_{1}+0.015 i_{2}+0.045 j, & \eta^{5}=0.913+0.008 i_{1}+0.017 i_{2}+0.062 j, \\
\eta^{6}=0.844+0.023 i_{1}+0.024 i_{2}+0.11 j, & \eta^{7}=0.87+0.025 i_{1}+0.027 i_{2}+0.079 j, \\
\eta^{8}=0.894+0.02 i_{1}+0.032 i_{2}+0.055 j
\end{array}
$$

The IDO correlation coefficients are computed and the fuzzy equivalence matrix is obtained by transitive closure calculation.

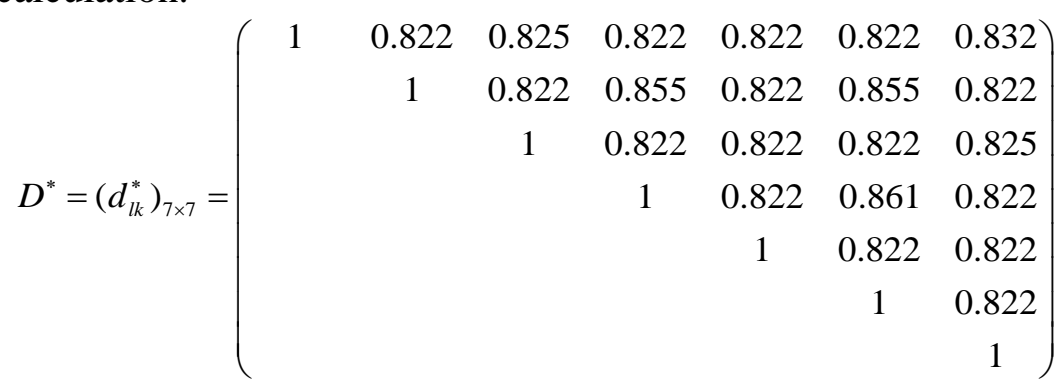

The selection $\lambda \in[0,1]$ is used to perform fuzzy clustering on each station.

When $\lambda=0.825$, the classification is $\left\{M_{2}, M_{4}, M_{8}\right\},\left\{M_{3}, M_{5}, M_{7}\right\},\left\{M_{4}, M_{8}\right\},\left\{M_{5}, M_{7}\right\}$; 
When $\lambda=0.85$, the classification is $\left\{M_{3}, M_{5}, M_{7}\right\},\left\{M_{5}, M_{7}\right\}$;

When $\lambda=0.86$, the classification is $\left\{M_{5}, M_{7}\right\}$.

From the clustering results, it can be seen that the stations $M_{5}$ and $M_{7}$ have the highest similarity with the reference subsystem. The similarity between the stations $M_{5}$ and $M_{7}$ and the reference subsystem is 0.9905 and 0.9883 , respectively. The influence of M5 and M7 on the weight of the reference subsystem $M_{1}$ is 0.501 and 0.499 respectively when the combination assignment method is used. Combined with the relevant safety check list criteria, from the set of $M_{5}, M_{7}$ can determine the site $M_{1}$ hydrazine fuel protection of the three indicators of weight.

Since $\mu$ is a quadratic relation degree, the $[0,1]$ is divided into three equal parts according to the principle of "sharing". The division points are from -0.333 to 0.333 , which are divided into $[-1,-0.333],(-0.333,0.333)$ and $[0.333,1]$ respectively. $\mu$ values fall in three intervals, respectively, reflecting the station system, "dangerous", "critical safety", "security" three security levels. Let $i_{1}=0.333, i_{2}=-0.333$, calculated $\mu_{1}=0.8418$, indicating that the station $M_{1}$ hydrazine fuel security level of security.

\section{Conclusion}

1) A safety evaluation model of four-station hydrazine fuel support based on Vague-SPA and fuzzy proximity degree is proposed. According to the characteristics of hydrazine fuel support, the safety evaluation index system was established. Based on the principle of fuzzy proximity, the comprehensive close degree of each scheme and positive and negative ideal scheme is calculated and the optimal solution of each index weight is obtained. The modified weights are applied to the SPA fuzzy clustering, and the weight of the third-level hydrazine fuel guarantee index of the reference station is determined by comparing the similar stations, and the safety evaluation grade of the station is obtained.

2) The differences of $i_{1}$ and $i_{2}$ reflect the different security states of the four-station hydrazine fuel support system, and also indicate the degree of conversion between the same, different and opposite, and give the quantitative State evolution.

3) The model makes use of the advantage of fuzzy information expressed by vague compound element to improve the traditional SPA method, which effectively overcomes the fuzziness of safety check list and the limitation of traditional SPA evaluation method, which provides a new way for safety evaluation of aviation four-station hydrazine fuel.

\section{References}

[1] ZHANG Yu, HUANG Zhi-yong. Application of Dow Chemical's Method in the Safety Assessment of Unsymmetrical Dimethyl Hydrazine Storage [J].Chemical Propellants \& Polymeric Materials,2014,12(1):86-89.

[2] LIU Bo, WANG Xuan-jun, HUANG Zhi-yong, et al. Research on Risk Evaluation Method of Unsymmetrical Dimethyl Hydrazine Storage Based on Grey Analytic Hierarchy Process [J]. Safety and Environmental Engineering, 2011, 18 (5):87-92.

[3] LIU Yuan, WANG Xuan-jun, XIA Ben-li, et al. Application of Fuzzy Overall Evaluation Method to Liquid Propellants Station Risk [J]. Safety and Environmental Engineering, 2010, 17(4):29-32.

[4] ZHANG Jun-hua, YANG Yao-hong, CHEN Nan-xiang. Application of Fuzzy Matter-element Model to Water Quality Evaluation of Reservoir[J]. Water Resources and Power, 2011, 29(1):17-19.

[5] ZHENG Xin, XU Kai-li, ZHOU Jia-hong. Research on the Safety Evaluation of the Tailing Pond by Way of the Set Pair Analysis[J]. Journal of Safety and Environment, 2008, 8(1):83-85.

[6]WEI De-cai, ZHANG Zheng-min, LI Chuang, et al.Airline Equipment Maintenance Safety 
Comprehensive Evaluation Based on Fuzzy Asymmetric Proximity and Entropy[J].China Safety Science Journal.2012,22(2):107-111.

[7] ZHU Ying-gui, YIN Guang-hui, YU Zhen-bing. Establishment and Application of Uncertain Multiple Attributive Operation Decision Based on Proximal Grade of Scheme [J]. Fire Control \& Command Control, 2014, 39(1):68-70. 